\title{
Ewald sum of the Rotne-Prager tensor
}

\author{
C. W. J. Beenakker \\ Philips Research Laboratories, 5600 JA Eindhoven, The Netherlands
}

(Received 2 April 1986; accepted 17 April 1986)

The lattice sum of the Rotne-Prager hydrodynamic mobility tensor is cast into a rapidly converging form by an Ewald summation technique. The result has a direct application to the problem of how to deal with the long range of hydrodynamic interactions in computer simulations of macromolecular solutions.

A widely used technique for dealing with long-ranged interactions in Monte Carlo or molecular dynamics calculations is to impose periodic boundary conditions on the cell containing the system (by translating the cell to fill the whole of space) and then to sum the interactions of that cell with each of its images. ${ }^{1}$ For computational purposes it is essential that this lattice sum is cast into a form which converges rapidly. For Coulomb interactions this is the wellknown Ewald sum. ${ }^{2}$ Hydrodynamic interactions, surprisingly enough, though having the same long range, have not been dealt with by this technique. Instead, truncations of these interactions have been proposed in the literature ${ }^{3,4}$; these suffer, however, from a slow convergence with increasing interaction range. A related approach ${ }^{5}$ has been to assume that hydrodynamic interactions in a suspension are effectively screened, with an empirically determined screening length. This assumption, however convenient it may be, is nonetheless physically incompatible with freely moving suspended particles. ${ }^{6}$ That the lattice summation technique has not yet been used in computer simulations of hydrodynamically interacting systems, can be ascribed to lack of an analogous Ewald sum for hydrodynamic mobility tensors. It is the purpose of the present note to provide such a formula for the long-ranged part of the two-sphere mobility tensor, which is the so-called Rotne-Prager ten6or. ${ }^{7}$

Consider a three-dimensional periodic lattice in which each unit cell (volume $V$, numbered by the index $l$ ) contains $N$ spherical particles (radius $a$, numbered by the index $i$ ). The lattice points are given by the vectors $\mathbf{r}_{l}$ and the particles have position vectors $\mathbf{R}_{l l}=\mathbf{R}_{l}+\mathbf{r}_{l}$. We denote the force on a particle by $\mathbf{F}$, and assume that the total force on the particles in a unit cell vanishes:

$$
\sum_{i=1}^{N} \mathbf{F}_{i}=0
$$

Now consider the lattice sum

$$
\mathbf{S}_{t_{0}}=\sum_{l}\left(\sum_{l=1}^{N} \mathbf{M}_{t_{0} l_{0}, l l} \cdot \mathbf{F}_{l}\right) \text {, }
$$

where $i_{0}$ is a given particle in cell $l_{0}$ and $\mathbf{M}$ is the RotnePrager mobility tensor,

$$
\begin{aligned}
\mathbf{M}_{t_{0} l_{0}, l}= & (6 \pi \eta a)^{-1}\left\{\frac{3}{4} a x^{-1}(\mathbf{1}+\hat{\mathbf{x}} \hat{\mathbf{x}})\right. \\
& \left.+\frac{1}{2} a^{3} x^{-3}(\mathbf{1}-3 \hat{\mathbf{x}} \hat{\mathbf{x}})\right\} \text { for }(i, l) \neq\left(i_{0}, l_{0}\right),
\end{aligned}
$$

$$
\mathbf{M}_{i_{0} l_{0}, r_{0} l_{0}}=(6 \pi \eta a)^{-1} \mathbf{1} .
$$

Here the vector $\mathbf{x}$ (with magnitude $x$ and direction $\hat{\mathbf{x}}=\mathbf{x} / x$ ) represents the separation vector $\mathbf{R}_{l l}-\mathbf{R}_{t_{0} l_{0}}$. The solvent viscosity is denoted by $\eta$, and $\mathbf{1}$ is the unit tensor. Because of the long range of the RP tensor, the series (2) converges only slowly. Using the Ewald summation technique, ${ }^{2}$ in the version of Nijboer and de Wette, ${ }^{8,9}$ this series can be rewritten into a rapidly converging form.

The final result (derived below) is given by

$$
\begin{aligned}
& 6 \pi \eta a \mathbf{S}_{t_{0}}=\left(1-6 \pi^{-1 / 2} \xi a+\frac{40}{3} \pi^{-1 / 2} \xi^{3} a^{3}\right) \mathbf{F}_{t_{0}} \\
& +\sum_{\substack{l \\
\mathbf{R}_{t} \neq \mathbf{R}_{t_{0} l_{0}}}} \sum_{l=1}^{N} \mathbf{M}^{(1)}\left(\mathbf{R}_{l l}-\mathbf{R}_{t_{0} l_{0}}\right) \cdot \mathbf{F}_{t} \\
& +V^{-1} \sum_{\substack{\lambda \\
\mathbf{k}_{\lambda} \neq 0}}^{N} \sum_{i=1}^{N} \mathbf{M}^{(2)}\left(\mathbf{k}_{\lambda}\right) \cdot \mathbf{F}_{\imath} \cos \left\{\mathbf{k}_{\lambda} \cdot\left(\mathbf{R}_{\iota}-\mathbf{R}_{\iota_{0}}\right)\right\}
\end{aligned}
$$

with the definitions

$$
\begin{aligned}
\mathbf{M}^{(1)}(\mathbf{r})= & \mathbf{1}\left\{\left(\frac{3}{4} a r^{-1}+\frac{1}{2} a^{3} r^{-3}\right) \operatorname{erfc}(\xi r)+\left(4 \xi^{7} a^{3} r^{4}+3 \xi^{3} a r^{2}-20 \xi^{5} a^{3} r^{2}-\frac{9}{2} \xi a+14 \xi^{3} a^{3}\right.\right. \\
& \left.\left.+\xi a^{3} r^{-2}\right) \pi^{-1 / 2} \exp \left(-\xi^{2} r^{2}\right)\right\}+\hat{\mathbf{r}} \hat{\mathbf{r}}\left\{\left(\frac{3}{4} a r^{-1}-\frac{3}{2} a^{3} r^{-3}\right) \operatorname{erfc}(\xi r)+\left(-4 \xi^{7} a^{3} r^{4}\right.\right. \\
& \left.\left.-3 \xi^{3} a r^{2}+16 \xi^{5} a^{3} r^{2}+\frac{3}{2} \xi a-2 \xi^{3} a^{3}-3 \xi a^{3} r^{-2}\right) \pi^{-1 / 2} \exp \left(-\xi^{2} r^{2}\right)\right\}, \\
\mathbf{M}^{(2)}(\mathbf{k})= & (\mathbf{1}-\hat{\mathbf{k}} \hat{\mathbf{k}})\left(a-\frac{1}{3} a^{3} k^{2}\right)\left(1+\frac{1}{4} \xi^{-2} k^{2}+\frac{1}{8} \xi^{-4} k^{4}\right) 6 \pi k^{-2} \exp \left(-\frac{1}{4} \xi^{-2} k^{2}\right) .
\end{aligned}
$$

The expression (4) consists of two lattice sums, one in real space over lattice vectors $\mathbf{r}_{l}$, and one in reciprocal space over reciprocal lattice vectors $\mathbf{k}_{\lambda}$ [ satisfying $\exp \left(i \mathbf{k}_{\lambda} \cdot \mathbf{r}_{l}\right)=1$ for all $l$ ]. The first series contains the complement of the error function,

$$
\operatorname{erfc}(x)=1-\operatorname{erf}(x)=2 \pi^{-1 / 2} \int_{x}^{\infty} \exp \left(-t^{2}\right) d t
$$


Both series converge exponentially fast, with the convergence rate controlled by the parameter $\xi>0$. For optimal convergence, $\xi$ should be chosen neither too small nor too large; $\xi=\pi^{1 / 2} V^{-1 / 3}$ is a good choice in the case of a simple cubic lattice.

To arrive at the result (4), we first note an alternative representation of the two-sphere RP tensor (3a):

$$
\begin{aligned}
6 \pi \eta a \mathbf{M}_{t_{0} l_{0}, l l}= & \left(\frac{3}{4} a+\frac{1}{4} a^{3} \nabla^{2}\right)\left(\nabla^{2} \mathbf{1}-\nabla \nabla\right)\left|\mathbf{R}_{t l}-\mathbf{R}_{t_{0} l_{0}}\right|, \\
& (i, l) \neq\left(i_{0}, l_{0}\right),
\end{aligned}
$$

where $\boldsymbol{\nabla}=\partial / \partial\left(\mathbf{R}_{l l}-\mathbf{R}_{t_{0} l_{0}}\right)$. We may therefore write

$$
\begin{aligned}
6 \pi \eta a \mathbf{M}_{t_{0} l_{0}, l l}= & \mathbf{M}^{(1)}\left(\mathbf{R}_{l l}-\mathbf{R}_{t_{0} l_{0}}\right) \\
& +\mathbf{M}^{(2)}\left(\mathbf{R}_{l l}-\mathbf{R}_{t_{0} l_{0}}\right), \\
& (i, l) \neq\left(i_{0}, l_{0}\right)
\end{aligned}
$$

with

$\mathbf{M}^{(1)}(\mathbf{r})=\left(\frac{3}{4} a+\frac{1}{4} a^{3} \nabla^{2}\right)\left(\nabla^{2} \mathbf{1}-\nabla \nabla\right)\{r \operatorname{erfc}(\xi r)\}$,

$\mathbf{M}^{(2)}(\mathbf{r})=\left(\frac{3}{4} a+\frac{1}{4} a^{3} \nabla^{2}\right)\left(\nabla^{2} \mathbf{1}-\nabla \nabla\right)\{r \operatorname{erf}(\xi r)\}$,

and $\xi>0$ an arbitrary parameter. We now substitute the decomposition (8) into Eq. (2),

$$
\begin{aligned}
6 \pi \eta a \mathbf{S}_{t_{0}}= & \mathbf{F}_{t_{0}}+\sum_{\substack{l \\
(l, l) \neq\left(t_{0}, l_{0}\right)}} \sum_{t=1}^{N} \mathbf{M}^{(1)}\left(\mathbf{R}_{l l}-\mathbf{R}_{t_{0} l_{0}}\right) \cdot \mathbf{F}_{l} \\
& -\mathbf{M}^{(2)}(\mathbf{r}=0) \cdot \mathbf{F}_{t_{0}} \\
& +\sum_{l} \sum_{t=1}^{N} \mathbf{M}^{(2)}\left(\mathbf{R}_{l l}-\mathbf{R}_{t_{0} l_{0}}\right) \cdot \mathbf{F}_{t} .
\end{aligned}
$$

The first series on the right-hand side of Eq. (11) is rapidly converging. The second series converges rapidly on the reciprocal lattice. The transformation to reciprocal space is performed by means of the formula ${ }^{8}$

$$
\sum_{l} g\left(\mathbf{r}_{l}\right)=V^{-1} \sum_{\lambda} g\left(\mathbf{k}_{\lambda}\right),
$$

with the Fourier transform of a function $g$ defined by

$$
g(\mathbf{k})=\int d \mathbf{r} e^{i \mathbf{k} \cdot r} g(\mathbf{r}) .
$$

We may therefore write

$$
\begin{aligned}
\sum_{l} \sum_{t=1}^{N} \mathbf{M}^{(2)}\left(\mathbf{R}_{l l}-\mathbf{R}_{t_{0} l_{0}}\right) \cdot \mathbf{F}_{t} \\
\quad=V^{-1} \sum_{\substack{\lambda \\
\mathbf{k}_{\lambda} \neq 0}} \sum_{t=1}^{N} e^{-i \mathbf{k}_{\lambda} \cdot\left(\mathbf{R}_{t}-\mathbf{R}_{\left.t t^{\prime}\right)}\right.} \mathbf{M}^{(2)}\left(\mathbf{k}_{\lambda}\right) \cdot \mathbf{F}_{t},
\end{aligned}
$$

where the terms with $\mathbf{k}_{\lambda}=0$ vanish by virtue of Eq. (1).

It remains to calculate $\mathbf{M}^{(1)}(\mathbf{r}), \quad \mathbf{M}^{(2)}(\mathbf{k})$, and $\mathbf{M}^{(2)}(\mathbf{r}=0)$. The expression (5) for $\mathbf{M}^{(1)}(\mathbf{r})$ follows straightforwardly upon carrying out the differentiations in Eq. (9). To obtain the Fourier transform of $\mathbf{M}^{(2)}(\mathbf{r})$ we perform partial integrations,

$$
\begin{aligned}
\mathbf{M}^{(2)}(\mathbf{k})= & -(\mathbf{1}-\hat{\mathbf{k}} \hat{\mathbf{k}})\left(a-\frac{1}{3} a^{3} k^{2}\right){ }_{4}^{\frac{3}{4}} k^{2} \int d \mathbf{r} e^{i \mathbf{k} \cdot r} r \operatorname{erf}(\xi r) \\
= & -(\mathbf{1}-\hat{\mathbf{k}} \hat{\mathbf{k}})\left(a-\frac{1}{3} a^{3} k^{2}\right) 3 \pi k \\
& \times \int_{0}^{\infty} d r r^{2} \sin k r \operatorname{erf}(\xi \mathrm{r}) .
\end{aligned}
$$

Evaluation of the remaining scalar integral ${ }^{10}$ yields Eq. (6). Finally $\mathbf{M}^{(2)}(\mathbf{r}=0)$ can be most conveniently calculated by integrating the Fourier transform,

$$
\begin{aligned}
& \mathbf{M}^{(2)}(\mathbf{r}=0)=(2 \pi)^{-3} \int d \mathbf{k} \mathbf{M}^{(2)}(\mathbf{k}) \\
& =1 \pi^{-1 / 2}\left(6 \xi a-\frac{40}{3} \xi^{3} a^{3}\right),
\end{aligned}
$$

where we have used the result (6).

This completes the derivation of the Ewald sum of the Rotne-Prager tensor. In dilute systems, where the assumption of pairwise additivity of the hydrodynamic interactions is justified, the RP tensor contains all the long-range contributions to the mobility: Corrections fall off at least as fast as the inverse fourth power of the interparticle separation, ${ }^{11}$ and therefore do not give rise to convergence problems. In more concentrated systems, to be sure, many-body hydrodynamic interactions have been shown to play an important role. $^{12}$ There is, nonetheless, theoretical and experimental evidence ${ }^{13,14}$ that to a certain extent these contributions may be accounted for through an effective pair mobility, which is just the RP tensor-but with the solvent viscosity replaced by the concentration-dependent effective viscosity of the suspension. As a final remark, we note that -although the above analysis was performed for a monodisperse solutionthe extension to a system with spherical particles of different radii $a_{l}(i=1,2, \ldots, N)$ is immediate ${ }^{11}$ : One need only replace in each of the above equations the radius $a$ to the first power by $a_{t_{0}}$, and the radius $a$ to the third power by $\frac{1}{2} a_{t_{0}}\left(a_{t_{0}}^{2}+a_{t}^{2}\right)$.

'S. G. Brush, H. L. Sahlin, and E. Teller, J. Chem. Phys. 45, 2102 (1966); R. O. Watts and I. J. McGee, Liquid State Chemical Physics (Wiley, New York, 1976).

${ }^{2}$ P. P. Ewald, Ann. Phys. 64, 253 (1921).

${ }^{3}$ W. van Megen, I. Snook, and P. N. Pusey, J. Chem. Phys. 78, 931 (1983). ${ }^{4}$ J. Bacon, E. Dickinson, and R. Parker, Faraday Discuss. Chem. Soc. 76, 165 (1983).

${ }^{5}$ I. Snook, W. van Megen, and R. J. A. Tough, J. Chem. Phys. 78, 5825 (1983).

${ }^{6}$ The point is that the screened mobility results from Darcy's equation for flow through a bed of particles which are immobilized by external forces and is a consequence of the absorption of fluid momentum at the particle surfaces. In this respect such porous media are fundamentally different from suspensions of freely moving particles which merely scatter the momentum flow.

${ }^{7}$ J. Rotne and S. Prager, J. Chem. Phys. 50, 4831 (1969).

${ }^{8}$ B. R. A. Nijboer and F. W. de Wette, Physica 23, 309 (1957).

${ }^{9}$ For a related application see R. Kapral and D. Bedeaux, Physica A 91, 590 (1978).

${ }^{10} \mathrm{To}$ evaluate this integral, write $\int_{0}^{\infty} d r r^{2} \sin k r \operatorname{erf}(\xi r)=-\left(\partial^{2} / \partial k^{2}\right)$

$\times \int_{0}^{\infty} d r \sin k r \operatorname{erf}(\xi r)$, where $\int_{0}^{\infty} d r \sin k r \operatorname{erf}(\xi r)=\int_{0}^{\infty} d r \sin k r$

$-\int_{0}^{\infty} d r \sin k r \operatorname{erfc}(\xi r)=k^{-1}-k^{-1}\left\{1-\exp \left(-\frac{1}{4} \xi^{-2} k^{2}\right)\right\}$.

${ }^{11}$ B. U. Felderhof, Physica A 89, 373 (1977).

${ }^{12}$ C. W. J. Beenakker and P. Mazur, Phys. Lett. A 91, 290 (1982).

${ }^{13}$ C. W. J. Beenakker and P. Mazur, Physica A 126, 349 (1984).

${ }^{14}$ C. W. J. Beenakker, Physica A 128, 48 (1984). 